\title{
Kinetic Models and Qualitative Abstraction for Relational Learning in Systems Biology
}

Gabriel Synnaeve, Katsumi Inoue, Andrei Doncescu, Taisuke Sato Yoshitaka Kameya, Masakazu Ishihata, Hidetomo Nabeshima 2009 internship at NII (Tokyo),
now at E-Motion@INRIA / Collège de France (Paris) January 27, 2011 


\section{Introduction}

Problems: exploit experimental data, learn what rules the cell.

Method: discretize metabolites concentration, combine with existing pathways structures, use kinetic models with inductive logic programming. 
(1) Previous works

- Metabolic Pathways

- Inductive Logic Programming

- Bibliography

A finer logic modeling

- The trap

- Kinetic modeling

- Implementation

- Ranked Results

- Where next? 
(1) Previous works

- Metabolic Pathways

- Inductive Logic Programming

- Bibliography

(2) A finer logic modeling

- The trap

- Kinetic modeling

- Implementation 
(1) Previous works

- Metabolic Pathways

- Inductive Logic Programming

- Bibliography

(2) A finer logic modeling

- The trap

- Kinetic modeling

- Implementation

(3) Results and further

- Ranked Results

- Where next? 


\section{(Metabolic) Pathways}

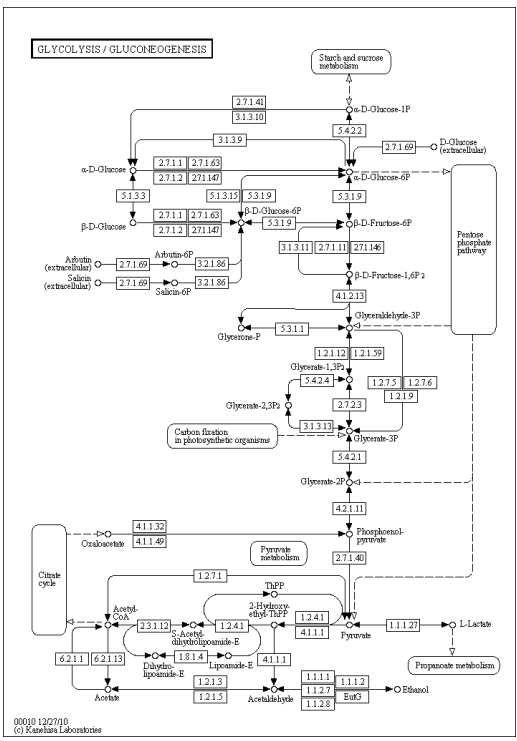

- Graphs of interconnected reactions

- Glucose enters ATP $\Rightarrow{ }_{A D P}$ G6P

- Chain of reactions to take energy and store it in ATP/NADH (2 per molecule of Glucose)

- Acetyl CoA is at the origin of the Krebs cycle (part of cellular respiration) 


\section{Glycolysis and Pentose Phosphate of E. Coli}

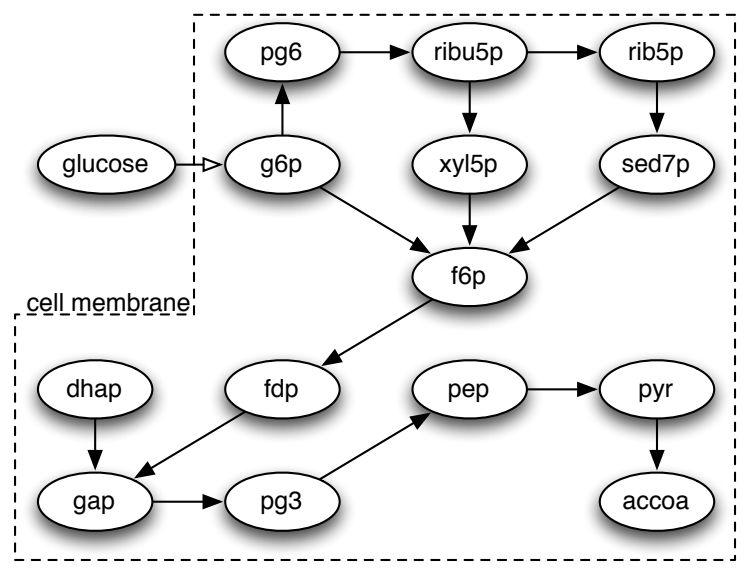




\section{Abduction \& Induction}

ILP strength lies in the fact that learnt rules/clauses are directly useable in a logical program.

\section{Induction \& Abduction}

From $\mathcal{B}$ ackground Knowledge $\wedge \mathcal{E}$ xamples

$\diamond$ Find $\mathcal{H}$ ypotheses satisfying $\mathcal{B} \wedge \mathcal{H}=\mathcal{E}$ and $\mathcal{B} \cup \mathcal{H} \not \models \perp$ Abduction: ground (or $\exists$ quant.) formulaes, direct causes of observations that are called explanations. Induction: universally $(\forall)$ quantified formulaes (small $\mathcal{B})$, more general hypotheses.

R. J. Mooney: Integrating abduction and induction in machine learning. IJCAl97 Workshop on Abduction and Induction in Al, 37-42 (1997).

Flach P. A., Kakas A. C.: Abduction and induction: Essays on their relation and integration. Kluwer (2000). 


\section{Inverse Entailment (Consequence Finding)}

ILP is interested in the formulas derived from $\mathcal{B} \wedge \neg \mathcal{E}$ that are not derived from $\mathcal{B}$ alone.

Inverse Entailment

The previous definition is equivalent to $\mathcal{B} \wedge \neg \mathcal{E} \mid \neg \neg \mathcal{H}$ and $\mathcal{B} \nvdash \neg \mathcal{H}$.

We can then use a consequence finding procedure (resolution, tableaux) to find $\neg \mathcal{H}$ (SOLAR).

Inoue, K.: Linear resolution for consequence finding. Artificial Intelligence 56:301-353 (1992).

清 Inoue K.: Induction as consequence finding. Machine Learning, 55:109-135 (2004).

河 Nabeshima H., Iwanuma K., and Inoue K.: SOLAR: A Consequence Finding System for Advanced Reasoning. TABLEAUX 2003, LNAI, Vol. 2796, pp. 257-263, Springer (2003). 


\section{New age began there}

The point for automatic qualitative reasoning through ILP has been made.

King, R., Whelan, K., Jones, F., Reiser, P., Bryant, C., Muggleton, S., Kell, D., and Olivier, S. (2004). Functional genomic hypothesis generation and experimentation by a robot scientist. Nature, 427:247-252.

囯 King, R., Garrett, S., and Coghill, G. (2005). On the use of qualitative reasoning to simulate and identify metabolic pathways. Bioinformatics, 21(9):2017-2026. 


\section{Inhibitionary effect of toxins}

Metabolic flux analysis through induction: rules that explain the concentration changes (up or down) between 2 experiments, with and w/o toxin.

Doncescu, A., Inoue K., Yamamoto Y.: Knowledge Based Discovery in Systems Biology Using CF-Induction. LNCS N.4570, pages 395-404 (2007). 


\section{Dealing with kinetics?}

Main complete models use ordinary differential equations.

Temporal logic combined with stochastic logic programming $\Rightarrow$ kinetic models.

Franco R. and Canela E.: Computer simulation of purine metabolism. Eu- ropean Journal of Biochemistry, 144:305-315 (1984).

言 Fages, F., Soliman, S., and France, I. R. (2008). Model revision from temporal logic properties in systems biology. In: Probabilistic Inductive Logic Programming. LNAI, volume 4911, pages 287-304. 


\section{Limits of The Previous Models}

- No models for dynamic transitions

- Not enough information to be precise enough:

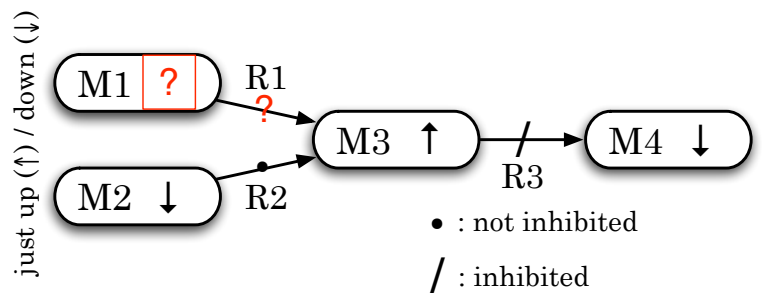




\section{Dealing With More Knowledge}

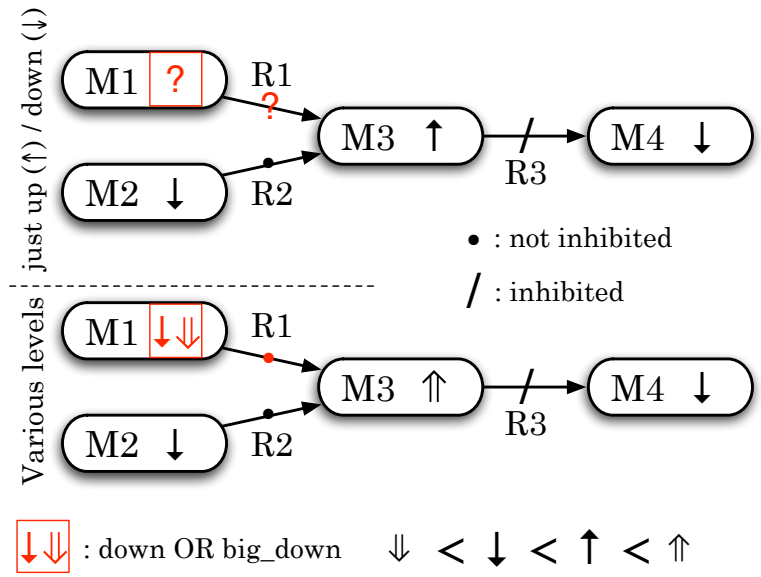




\section{Be More Precise but Avoid Overfitting}

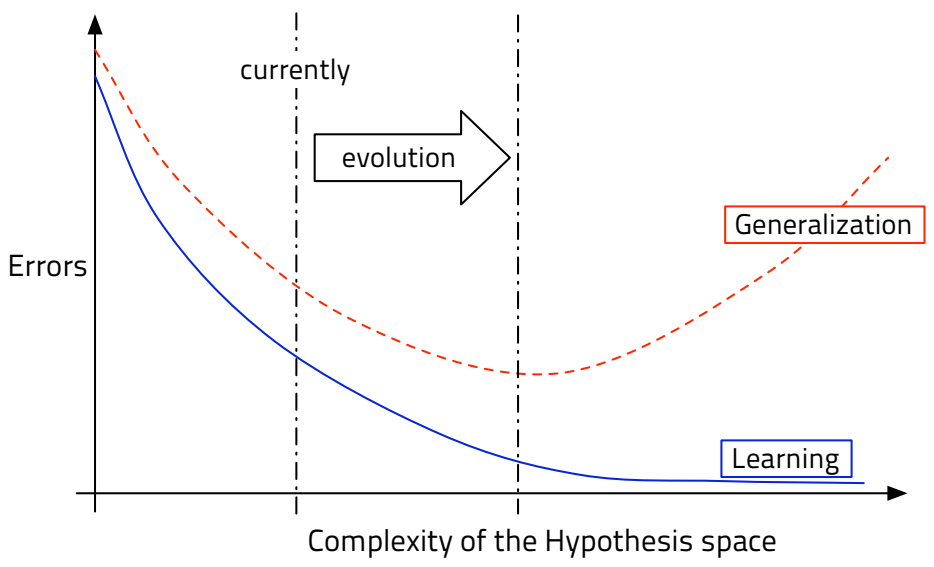




\section{Michaelis-Menten Kinetics}

\section{Speed of a one-way reaction}

$v=\#\{$ products per second per mole of the enzyme $\}$

$s S \rightarrow p P \Rightarrow v=-\frac{1}{s} \frac{d[S]}{d t}=\frac{1}{p} \frac{d[P]}{d t}$

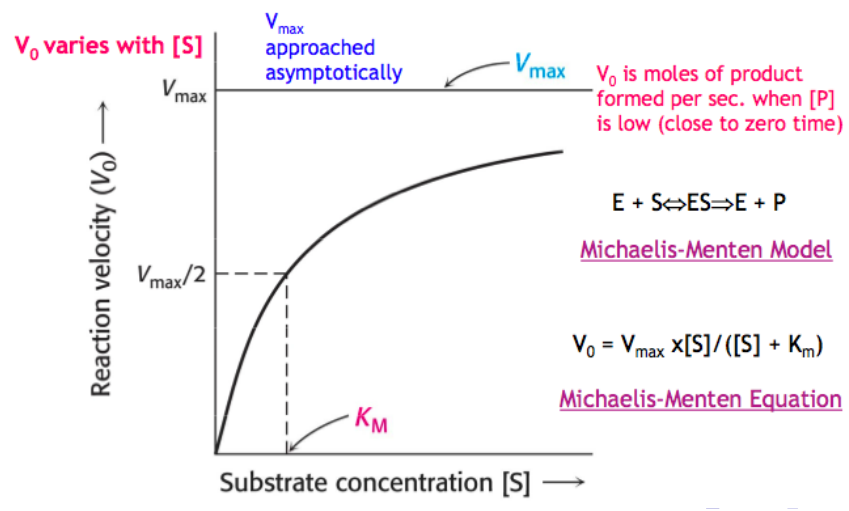




\section{Simplification of Michaelis-Menten Equation}

$$
E+S \leftrightharpoons \leftrightharpoons_{k_{-1}}^{k_{1}} E S \rightarrow^{k_{2}} E+P
$$

Michaelis - Menten equation : $\frac{d[P]}{d t}=V_{m} \frac{[S]}{[S]+K_{m}}$

$$
\frac{d[P]}{d t} \longrightarrow \text { disc.time } \frac{[P]_{T+\text { timestep }}-[P]_{T}}{(T+\text { timestep })-T}
$$

$$
\text { (1) and (2) } \Rightarrow V_{m} \frac{[S]_{T}}{[S]_{T}+K_{m}} \approx \frac{[P]_{T+\text { timestep }}-[P]_{T}}{(T+\text { timestep })-T}
$$

We chose to work with a constant timestep :

$$
\Rightarrow[P]_{T+1}=V_{m} \frac{[S]_{T}}{[S]_{T}+K_{m}}+[P]_{T}
$$




\section{Additional work and tools}

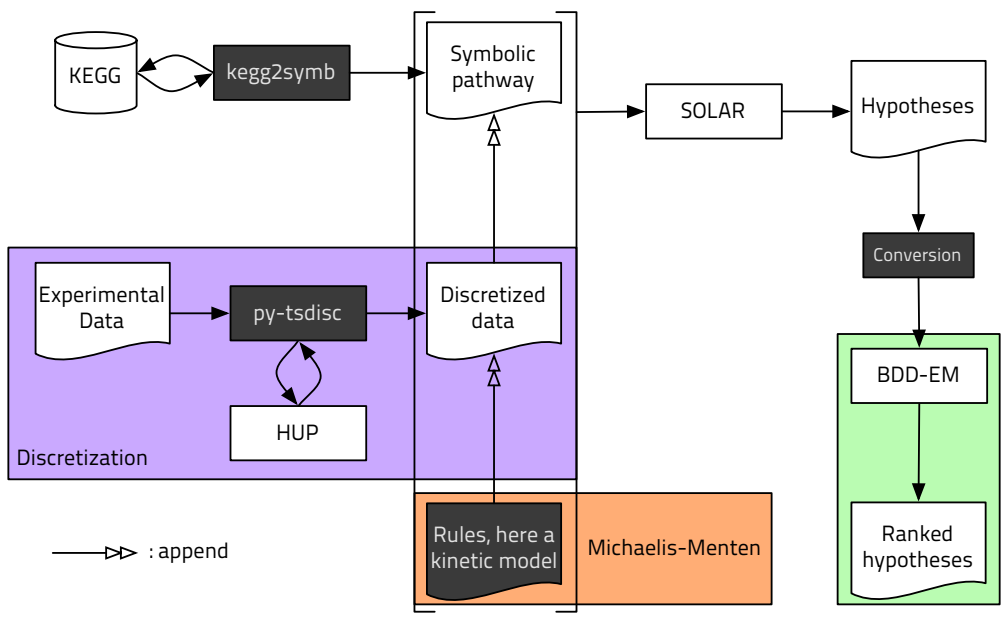




\section{HUP: HMM Utility Program}

Clustering method that uses

Continuous Hidden Markov Model + Bayesian Score:
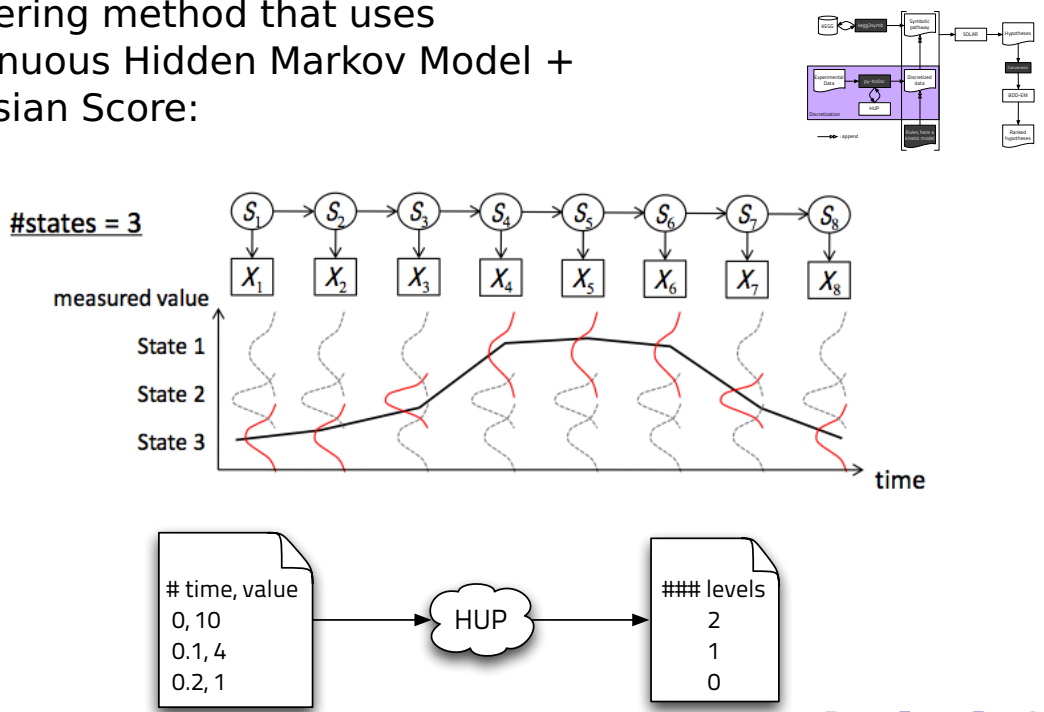


\section{Wrapping HUP}
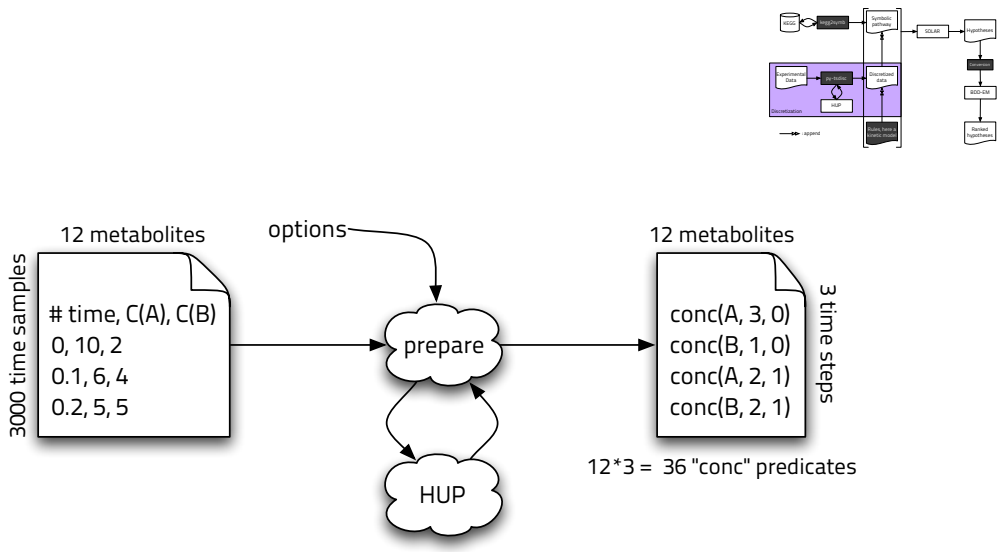


\section{Logical Kinetic Modeling}

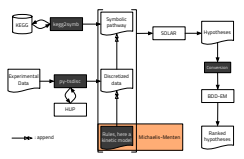

Approximations for extreme values in:

$$
[P]_{T+1}=V_{m} \frac{[S]_{T}}{[S]_{T}+K_{m}}+[P]_{T}
$$




\section{Logical Kinetic Modeling (rules)}

$$
[S] \ll K_{m}
$$

reaction $(S, P, K m) \wedge$ concentration $(S, 0,0) \wedge$ concentration $(\mathrm{Km}, 2,0) \wedge$ concentration $(\mathrm{P}, \mathrm{L}, 0) \rightarrow$ concentration $(P, L, 1)$

$$
[S] \simeq K_{m}
$$

reaction $(S, P, K m) \wedge$ concentration $(S, 1,0) \wedge$ concentration $(\mathrm{Km}, 1,0) \wedge$ concentration $(P, L, 0) \rightarrow$ concentration $(P, L, 1)$

$$
[S] \gg K_{m}
$$

reaction $(S, P, K m) \wedge$ concentration $(S, 2,0) \wedge$ concentration $(\mathrm{Km}, 0,0) \rightarrow$ concentration $(P, 2,1)$ 


\section{Data-centric schema}

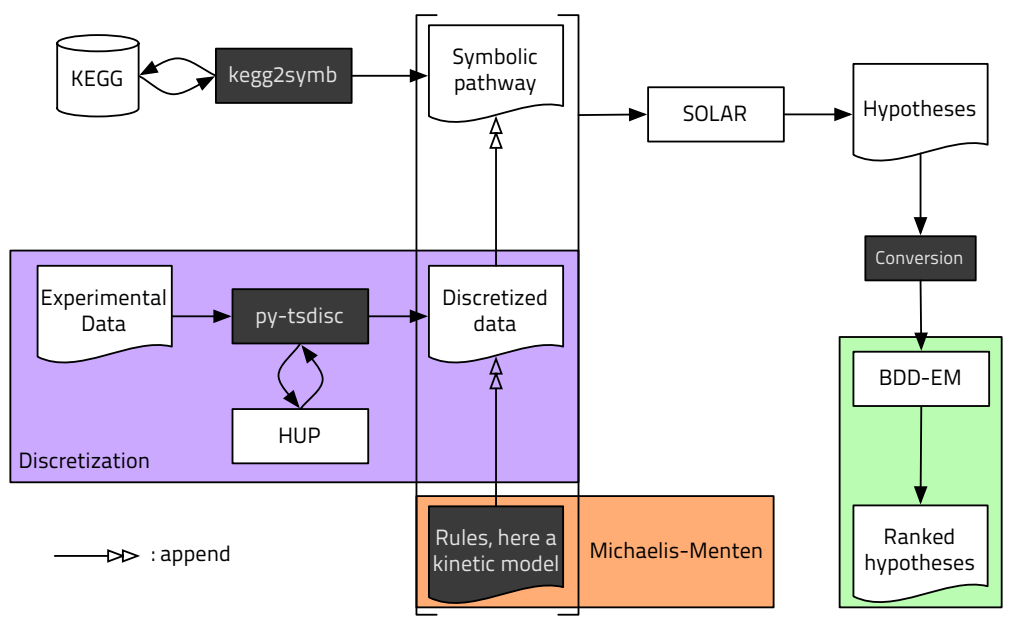




\section{Inputs}

Structure of the pathway(s) + Background knowledge $(\mathrm{MM})+$ Metabolites concentrations

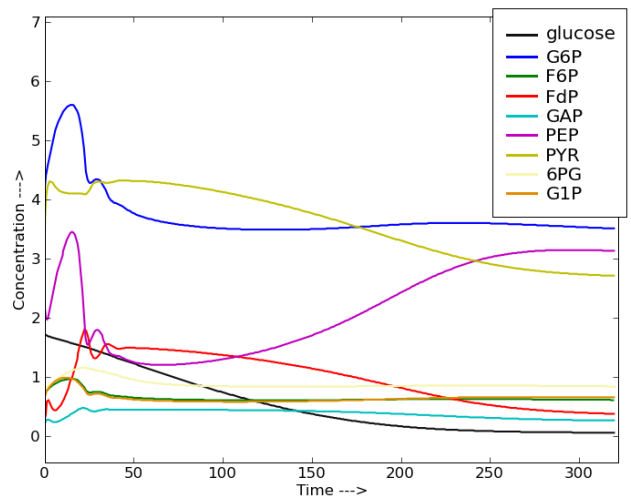




\section{Ranking the hypotheses with BDD-EM}

\begin{tabular}{l|l|l} 
Hyp. no. & Probability & Abducted concentrations levels at $\mathrm{T}=0$
\end{tabular}

H130

$\mathrm{H} 392$

$\mathrm{H} 216$

$\mathrm{H} 196$

H356

H94

$\mathrm{H} 251$

$\mathrm{H} 286$

$\mathrm{H} 405$

$\mathrm{H} 167$

H378

pg3: 2, adp: 0

sed7p: 0, e4p: 2, f6p: 0, pg3: 2, adp: 0

pg3: 2, adp: 0, pep: 0, atp: 2, pyr: 2

fdp: 0, dhap: 2, gap: 0, pg3: 2, adp: 0

pg3: 2, adp: 0, g6p: 1, nadph: 1

sed7p: 0, e4p: 2, f6p: 0, pg3: 2, adp: 0, pep: 0, atp: 2, pyr: 2

3.497. $E^{-2}$ glucose: 2, adp: 0, pg3: 2

3. $382 . E^{-2}$

2.796. $E^{-2}$

2.743. $E^{-2}$

sed7p: 0, e4p: 2, f6p: 0, fdp: 0, dhap: 2 , gap: 0, pg3: 2, adp: 0

pg3: 2, adp: 0, pep: 2, atp: 0

sed7p: 0, e4p: 2, f6p: 0, pg3: 2, adp: 0, g6p: 1 , nadph: 1

$1.974 . E^{-8}$ glucose: $2, \operatorname{adp}: 0, \operatorname{sed} 7 p: 0$, e4p: 2, f6p: 0 , fdp: 0, dhap: 2, gap: 0, pg3: 2, pep: 0, atp: 2 , pyr: 2, g6p: 0, nadph: 2, pg6: 1 


\section{Is is correct?}

Ok with: glucose $+2 \mathrm{ADP}+2 \mathrm{P}+2 \mathrm{NAD}^{+} \rightarrow$

2 pyruvate $+2 \mathrm{ATP}+2\left(\mathrm{NADH}, \mathrm{H}^{+}\right)+2 \mathrm{H}_{2} \mathrm{O}$

Agree with:

圆 Peters-Wendisch, P., Schiel, B., Wendisch, V., and et al., E. K. (2001).Pyruvate carboxylase is a major bottleneck for glutamate and lysine production by corynebacterium glutamicum. Molecular Microbiol. Biotechnol., 3(2). 


\section{Full system}

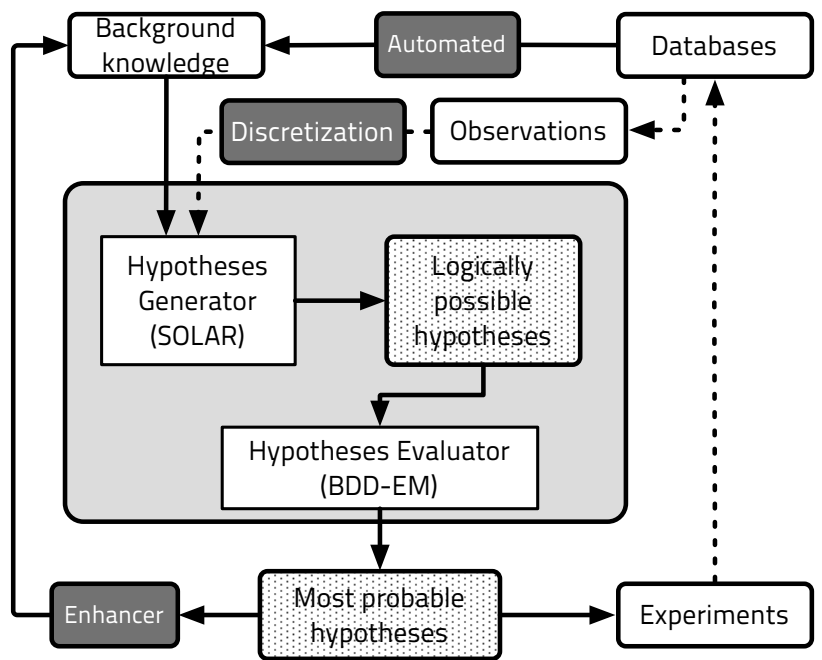




\section{Future tracks:}

- Enhancing of the knowledge base, 2 simple and sound algorithms (one in the paper):

- most probable hypotheses first

- smallest number of hypothesis additions (biggest abducibles coverage first)

- Finer discretization: trivial with our continuous HMMs with parameter tying.

- Automatic generation of MM rules (for orders > 3). 


\section{Conclusion}

We presented and validated a method and tools to work on real data.

Working with other experiments on more complex organisms and pathways (for instance S. Ce) will require:

- Enhancing of the KB

- Finer discretization

- Kinetic rules 


\section{Thanks}

Thank you for your attention. 


\section{Any questions?}

\section{Any questions?}

Me: gabriel.synnaeve@gmail.com Prof. Inoue: ki@nii.ac.jp 